Meta

Journal des traducteurs

Translators' Journal

\title{
Éditologie : une sociolinguistique de la science
}

\section{Jean Claude Baudet}

Volume 40, numéro 2, juin 1995

Usages sociaux des termes : théories et terrains

URI : https://id.erudit.org/iderudit/002001ar

DOI : https://doi.org/10.7202/002001ar

Aller au sommaire du numéro

Éditeur(s)

Les Presses de l'Université de Montréal

ISSN

0026-0452 (imprimé)

1492-1421 (numérique)

Découvrir la revue

Citer cet article

Baudet, J. (1995). Éditologie : une sociolinguistique de la science. Meta, 40(2),

216-223. https://doi.org/10.7202/002001ar

\section{Résumé de l'article}

Une nouvelle approche du problème épistémologique est proposée, basée sur une définition de la science considéré comme " un ensemble de textes édités ". Les textes étant des ensembles de termes, l'épistémologie se divise en deux parties : l'étude des termes (terminologie) et l'étude de l'édition (éditologie). En insistant sur les conditions sociales du processus d'édition, l'editologie est en fait une analyse sociolinguistique de la science. 


\title{
ÉDITOLOGIE: UNE SOCIOLINGUISTIQUE DE LA SCIENCE
}

\author{
JEAN CI.ALIDE BALIDET
}

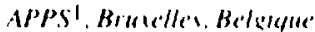

\begin{abstract}
Résumé

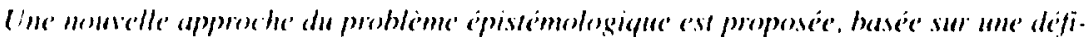

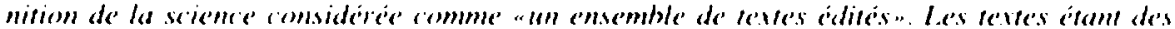

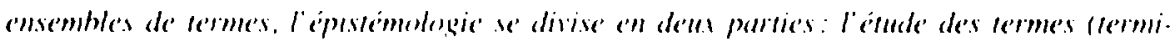

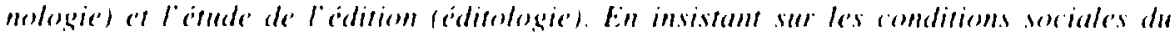

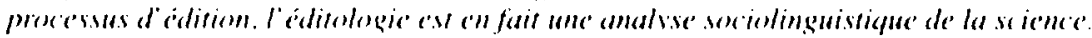

Il y a maintenant dix ans que j’ai introduit le mot éditologie dans le champ de l'épistémologie, pour désigner à la fois un domaine d'investigation el une méthode. "est en effet le 11 février 1984 que jorganisais, à Bruxelles, en tant que président d'APPS, un colloque sur l'éditologie des sciences industrielles, sous le haut patronage du ministre de l'Éducation nationale (de Belgique) de l'époyue, M. André Bertouille (Allard l984). Parce que l'éditologie peut être comprise comme un effort pour prendre en compte les données de la linguistique au profit de l'épistémologie, parce que l'éditologie pourra à son tour apporter à la linguistique des éléments de réflexion susceptibles de l'aider à progresser au moins dans deux directions importantes de la recherche contemporaine la lerminologie et la sémiologie -... et parce qu'enfin. l'éditologie est elle-même un discours, cest-à-dire une suite de termes justiciable d'ailleurs d'une analyse linguistique. il nous paraît utile de tenter ici de donner un exposé sur le développement et l'état actuel de notre recherche. Nous nous efforcerons de montrer spécialement les liens qui unissent l'éditologie à la linguistique, et plus spécialement à la sociolinguistique (Baudet |99|). Il ne s"agit donc pas de traiter de l'ensemble de la problématique que l'éditologie est en mesure d'éclairer, ce qui nécessiteratit de plus longs développements.

\section{DÉFINITION}

Le mot éditologie, par sa construction même. suggère une définition évidente: la science de l'édition. C'est en 1982 qu'un profiesseur de la Faculté de médecine de Nancy. Roger Bénichoux, a créé ce néologisme, lançant une publication périodique polycopiéc. Éditologice. Mais M. Bénichoux n'élait animé par aucune intention épistémologique. c'està-dire critique. Bien au contraire, acceptant la science comme elle est (c'est-à-dire acceptant les mécanismes institutionnels de production et de mise en circulation des savoirs: universités. revues. CNRS...). l’inventeur de cè néologisme voulait aider les chercheurs à mieux participer atu développement d'une science nullement remise en question (Bénichoux a' al. 1985). Pour notre auteur. en effel. l'éditologic signifiait simplement un ensemble de conseils (d'ailleurs effectivement fort utiles) destinés à publier, à éditer mieux. Expliquer aux jeunes chercheurs comment élablir une bibliographie, leur montrer comment rédiger la description d'un protocole expérimental, leur expliquer comment illustrer un article. voilà quelles étaient les ambitions de cette éditologic.

Le terme était done disponible, et il nous at paru particulièrement approprié pour désigner le type d'épistémologie que nous essityions à l'époxpue de construire. l éditologie

Mita. XI. 2. /495 
esi donc bien pour nous, en effet, l'étude de l'édition. mais à la double condition de prendre le mot étude dans son sens le plus large d'investigation critique (au sens kantien). et de prendre le mot édition dans son sens le plus restreint de mécanisme social de mise à la disposition des savoirs de la communaulé scientifique.

Dans les années 1980. l'épistémologie était traversée par de nombreux courants qui avaient presque tous, pour aussi variés que soient leurs soubassements idéologiques, en commun de prendre "la Science» - l'objet de l'épistémologie - comme un objet donné. déjà là, qu’il s’agissait de comprendre (analyser la méthode scientifique, retracer les grandes étapes du progrès scientifique...) sans mettre en cause les aspects sociaux de sa production. Même l'épistémologie marxiste, qui aurait pourtant dû réserver une altention particulière au caractère social du travail scientifique, réservail une place privilégiée à la science au sein des différents éléments constituant la "superstructure" des sociétés humaines, sans doute à cause de la prétention de scientificité du marxisme. Une critique radicale de la science ou hién était considéréc comme une hérésie par les divers rationalismes (marxisme compris), ou bièn était empêchée par l'absence de référence axiologique à mettre à la place de la science adétrônée" (science conçue comme seule détentrice. sinon de "la vérite", au moins de la méthode pour l'alteindre). C"est comme si les épislémologues - parce qu'étant eux-mêmes des scientifiques - refoulaient dans leur inconscient la nature sociale de la science. Il y avait certes le travail des sociologues de la connaissance? mais leurs résultats appartenant au champ de la sociologie étaient assez. peu connus des épistémologues. el d'ailleurs il ne s'agissait pas d'une véritable remise en cause, puisque la sociologie de la connaissance admettait loujours, au moins implicitement, la supériorité du mode de connaissance scientifique sur les autres modes de savoir.

Cependant. de l’école de l’épistémologie génétique (Jean Piaget) venait l'idée d’une relation forte entre connaissance el communication. et d'autre part l'histoire des sciences aboutissait à prendre conscience du caractère socialement construit des théories scientifiques. Bien plus, nous y reviendrons. l'histoire des sciences commençait à élargir son horizon aux techniques, ce qui eut pour conséquence que la prise en compte de l'évolution des techniques dans une perspective épistémologique (le fameux problème de la relation entre science "pure» et science "appliquée») induisit une réévaluation de la technique par rapport à la science (et donc par rapport à l'ensemble de la société, puisqu il apparaissait clairement désormais que l'opposition classique entre science et technique n était que la transposition de l'opposition soxiale entre classes oisives et classes laborieuses). Cette réévaluation nous paraît mériter le nom de véritable révolution copernicienne. surtout du fait de ses implications dans l'organisation sociale.

C'est donc en tentant de synthétiser les apports de la sociologie de la connaissance. de l'épistémologie génétique. de l'histoire des sciences et de l'histoire des techniques qu’il nous a paru nécessaire d'abord d'élucider le rapport, à nos yeux essentiel dans le sens. philosophique du terme essence. entre science et technique (Baudet 1978), et ensuite de disposer d'une définition de la science yui nous permît d'en essayer une critique. Cela nous amena d'une part à proposer le concept de STI (science-technique-industrie) comme objet réel de l'épistémologie. et en même temps (lès deux idées sont inséparablement liées) de mettre en évidence l'édition à la fois comme mécanisme social de production des savoirs et comme londement de la "scientificité" (Baudet 199()a). Nous donnerons donc de l'éditologie la définition plus complète suivante: étude épistémologique de l'édition des savoirs scientifiques, techniques et industriels, qui prend en compte la nature sociale (c'est-à-dire communicationnelle) des mécanismes de leur production et de leur validation. Le mot "communicationnelle» est là pour nous montrer qu'il s'agira bien. tinalement, de linguistique. Et d'autre part. la linguistique appartenant à la STI, elle serait susceptible elle-même d'une analyse éditologique, mais ceci sort du cadre que nous nous sommes fixé. 


\section{ÉDITOLOGIE ET TERMINOL.OGIE}

Le point de départ de notre épistémologie est de tenter de trouver une définition de la science qui permeltrait de distinguer un fait «scientifique" de toute autre production culturelle qui ne mériterail pas d'être qualifiée de uscientifique». Il nous a paru que l'on ne pourrait pas s'opposer à une définition qui commencerait par aftimner que la science est un ensemble de textes, c'est-à-dire finalement de termes. I a science est un discours. c'est-à-dire une production linguistique (ce qui, par exemple, écarte clairement de la scientificité des éléments de la culture comme les arts plastiques ou les comportements mondains). Plus précisément. et bien sûr la circularité de la détinition ne nous échappe pas, la science est l'ensemble de tous les textes... scientifiques (Baudet 1992). Cette proposition qui peut paraître naïve est déjà le résultat d’une observation sérieuse de la vie scientifique. qu il s'agisse de la vie actuelle des laboratoires (la synchronie) ou de l'histoire des sciences. des techniques et de l’industrie (la diachronie). Dès que l'on a vécu le quotidien d'un chercheur (et dans quelque discipline que ee soit), on sait que la préoccupation majeure - le but même de loule activité - est la publication. Les travaux de laboratoire les plus patients du chimiste ou du psychologue, les calculs les plus difficiles de l'astronome ou de l'économiste. les enquêtes les plus raffinées du sociologue ou du linguiste n ont finalement qu un aboutissement: la publication. Lat science est ainsi la transmission de textes de génération en génération: la communication est bien l'essentiel. Ft la communication au sein d'unc communatuté spécialisée. car bien sûr l'art ou la religion ou la littérature sont aussi communication. mais il y at une relation d'un tout autre ordre entre l'artiste et son public qu entre un mathématicien qui lit un "papier» dans un colloque et son auditoire formé d'autres mathématiciens. Ajoutons que la détinition éditologique de la science a pour nous le mérite de constituer ainsi l'objet science' en un observable. La délinition classique de la science (par exemple: ensemble de connaissances et de recherches ayant un degré suffisant d'unité et de généralité, qui est la formulation du lócahularé de la philosophie de Lalande) renvoie à des notions elles-mêmes problématiques (connaissance. unité. généralité...) et ne permét pas la délimitation concrète du champ d'études de l'épistémologie.

Si donc la science est un ensemble de textes. c'est surtout un ensemble de textes édités, c'est-à-dire que c'est dans les particularités du mécanisme d'édition que résidera la "scientificité". Nimporte quel texte (un roman. un règlement. une lettre privée...) est éventuellement édité, mais seuls les textes scientifiques subissent un mécanisme d'édition particulier, et qui fonde la "valeur" du texte. C"est en étudiant ce mécanisme que d'une part on assigne à l'épisfémologie un programme en deux parties - la terminologie pour l'étude critique des termes el l'éditologie pour celle de l'édition. puisque termes et édition sont les deux éléments constitutifs de la science - et que d’atre part on découvre que la science est sociale. Si les recherches de Newton ou d'Einstein sont qualifiées de sciemifiques. c'est parce que Newton èt Einstein ont publié des textes reconnus comme faisant partic de la physique par l'ensemble des physiciens. Et il appartiendra à l'éditologie de déterminer si les critères permettant cette reconnaissance sont liés aux termes (par exemple. le fait que les ouvrages de Newton ont été écrits en latin: ou encore le fait d'y trouver des termes comme masse, accélération, trajectoire...) ou a l'édition (par exemple le fait que les articles d'Ëinstein parurent dans des revues spécialiséess).

\section{DF. LA FAI.SIFIAHILITE A I,EIDTION}

L’importance des travaux de Karl Popper pour l'épistémologie contemporaine est bien connue. Si l'on examine son critere de la lalsifiabilite en quittant le domaine de la théorie pure pour revenir au concret. la dualité éditologique-terminologique de l'épistémologie 
devient une évidence. On trouve d’ailleurs, chez le philosophe autrichien lui-même, des phrases qui sont comme la préfiguration de l'éditologie. Quand il écrit que al'objectivité des énoncés scientifiques réside dans le fait qu'ils peuvent être intersubjectivement soumis a des tests" (Popper 1973: 41 ), quand il écril que "Comme toutes les représentations linguistiques [les théories scientifiques] sont des systèmes de signes ou de symboles" (Popper 197.3: 57), l’inventeur de la falsifiabilité ne fait rien d'autre qu affirmer que la science est un langage (une langure bie'll faite, selon l'expression célèbre), el que les tests sont loccasion d'une rencontre intersubjective: la science est linguistique et sociale. Mais il faut aller plus loin que dire que l'on peut faire des tests. C'est ici l'aboutissement de la pensée popperienne. Mais l'éditologie poursuit la réflexion êt l'observation au-delà, dans le concret des laboratoires: comment ces lests seront-ils organisés, par qui. comment les résultats éventuels seront-ils portés à la connaissance de la communauté scientifique? Ces tests sont d'ahord décrits (termes). ces descriptions sont éditées sous forme darticles dans des revues, ces articles sont lus (c’est-à-dire interprétés), des expériences sont menées, et leurs résultats, à leur tour, sont transcrits en textes (termes). édités. lus... C'est hien une communication. Il y a hien un émetteur. un récepteur, un message, un code... Et c'est bien. finalement, dans les mécanismes de cette édition que réside le critère réel de la scientificité.

C'est parce que la communauté scientifique reconnaît ces articles comme scientifiques, ces revues comme scientifique's, que nous pouvoms dire qu'il s'agit bien de science. Une thécoric policière, à propos d"un assassinat, est également falsifiable. C'est la méthode de la reconstitution du crime qui joue ici le rôle du test. Mais l'activité policière n'est pas une science (même si. justentent, il y a des relations évidentes entre la méthode scientifique et les méthodes de la police). Si une théorie doit être falsifiable pour être scientifique. cette condition nécessaire n'est pas encore suffisante. C"est dans la manière dont s'organisent socialement (communicationnellement) les procédures de test que l'on trouvera le vrai critère de la scientificicí.

L'éditologice, si elle prend en compte les résultats de l'épistémologie popperienne. veut aller au-delà de celle-ci dans deux directions. Dune part elle accepte de passer à l'examen des réalités soxiales. et examinera les comportements de la communauté scientifique. Less comportements généralement blâmés par celte communauté sont bien sûr parmi les plus intéressants: plagiat (un auteur utilise des travaux d'autres chercheurs sans en citer les sources, s'en attribuant implicitement la paternité). fausses publications (publier des expériences qui n'ont pas été faites, ou en falsifier les résultats), despotisme éditorial (un patron de laboratoire qui régente les publications de ses collaborateurs: un directeur de revue qui refuse certains articles pour des raisons extra-scientificues). Il y a ainsi toute une séric de perversions éditoriales que l'éditologie étudie avec profit. Le résultat le plus immédiat de ce type de recherche est de découvrir que dans certains cas les motivations du chercheur qui publie sont davantage d'ordre personnel (carriérisme) que d'ordre purement scientifique faire proseressis' la science').

D autre part, l'éditologie accepte également de passer de l'étude de la transmission et de la validation des savoirs a celle (linalement bien plus cruciale) de leur production même. ("est le sens de la complémentarité éditologie-terminologie: pour nous, l'étude des termes (de la science, mais en fait aussi des termes de n'importe quel discours) est indissolublement liè à l'étude de leur édition. "est une des données les mieux établies et les plus fécondes de la sociolinguistique davoir montré que les conditions sociales d'utilisation d'une langue (ses fonctions sociales) influençaient sa forme même (tant en ce qui concerne le lexique qu'en ce qui concerne les caractéristiques phonétiques, morphologiques et syntaxiques), et il fallat exploiter ce résultat dans l'analyse du discours scientifique. Mais l'étude des termes, c'est l'étude des concepts auxquels ils renvoient, el notre épistémologie pose que les conditions sociales vécues par l'homme de science 
modèlent sa créativité. c'est-à-dire la manière dont il ordonne les termes dont il dispose pour construire ses théories. L'exemple est banal mais éclairant: au XIX` siècle, les scientifiques créaient des néologismes à partir des racines grecques. aujourd hui ils utilisent les ressources de l'anglais courant. Etudier les termes et les concepts, tenter de comprendre. en partant du terme et de sa position dans un texte édité. comment naissent les idées nouvelles dans l'histoire de la science, voilà donc bien la mission difficile de l'éditologie. C'est ce que refusait Popper (1973: 26):

Je dois edarecir la distinction qui s impose entre la psychologie de la connaissance, qui traite des faits empiriques. ef la logique de la commaissance, que concernent les seules relations logigues |... | le travail du savant consiste al avancer des theories et a les soumelter a des lests. le vade initial. cet acte de concevoir ou d'inventer une theorie. ne me semble pas requerir une analyse logique ni même ètre susceptible d'en être l'objel. la question de savoir comment une idé nouvelle peut naître daus lesprit d un homme |... l ne releve pas de lanalya logique de la comnaissance scientifique $\mid . .$. | y il s'agit des processus impliques dans la stimulation el le janllissement d'une inspiration, je refuse de considérer leur reconstruction comme la tache de la logique de lat connaissinte.

Comment une idéc nouvelle naî dans l'esprit d'un homme nous imtéresse au plus hatu point: cette idée deviendra un terme (l'oxygène ches lavoisier quand il découvre les incohérences de la théorie du phlogistique, les quarks de Murray Gell-Mann quand il élatrore une nouvelle systématique des particules élémentaires...). el ce terme sera inséré dans un texte qui sera édité. qui sera done partagé par la communauté scientifique. De l'idée nouvelle au terme (question sémantique), du terme a la propagation de l'idée nouvelle (question pragmatique), il y a un double probleme que l'éditologie ne refuse pas de considérer. Et nous posons que ce double probleme est de nature sociolinguistique.

\section{DES MOTS AUX TERMES}

Quand Jacques lacan nous dit que l'inconscient est structuré comme un langage. il reformule la psychanalyse, critique de l'inconscient. Semblablement, l'éditologie reconnaît que les textes scientiliques (outre leurs caractéristiques d'édition) ont leur propre structure (qui se surajoute à la structure proprement linguistique de la langue naturelle utilisée). et reformule l'épistémologie. critique de la science. Bien sûr. il y a des termes te'tmique's dans les arts, dans le droit, dans la littérature... Mais il n'y a qu en science (c'est-à-dire dans le domaine de la STl que les termes fonctionnent vraiment en système. Et surtout, il n'y a qu'en science que ces termes sont agissants. François Dagognet (1969) a fort bien étudié ce qu'il appelle les comrespondances voro-strueturales de la chimie contemporaine, depuis la nomenclature de Lavoisier jusqu aux fommles de la chimie organique en passant par le fameux tableau de Mendeleiev. Il a également montré dans les domaines de la phytographie. de lat /oologie el de la nosologie que ce'est la création même d'un système de termes qui, chaque fois, a permis de maîtriser scientifiquement un donné surabondant (Dagognet 1970). Les termes ne sont pas inventés pour développer la science, comme une espéce de complément de linition. ("est l'invention même des termes qui constitue le mouvement scientifique avec, simultanément, la publication de ces termes. Quand le chimiste crée les termes sulfate, sulfite, sulfure, sur le modèle de chlorite, chlorite, chlorure. il ne crée pas simplement quelques mots commodes pour désigner les contenus de sess flacons. Il résume toute une théorie. Le rôle de l'oxygène dans la production des acides est résumé dans la suite des désinences ate, ite, ure. Il s'agit bien d un système nomenclatural (contrairement à la nomenclature non systématique et par cela même préscientifique des alchimistes), dans lequel chaque terne renvoie à tous les autres: sulf-renvoie à tous les composés contenant du soufre, -ate à tous less sels fortement oxygénés... Bien sûr. 
cette nomenclature, résultat d'un processus historique (éditologique: on peut citer et dater les ouvrages depuis Louis Guyton de Morveau, Antoine Laurent de Lavoisier, jusqu'aux publications actuelles de I'IUPAC. Intermational Union for Pure and Applied Chemistry). comporte des exceptions, des insuffisances nombreuses. Mais l'essentiel est qu'il s'agit bien d'un langage construit. et validé par l'édition.

Ce n'est que très récemment, dans l'histoire de la science, que le caractère agissant des termes scientifiques est clairement apparu. Avec l'avènement du génie logiciel, on assiste en effet à la création de langues artificielles qui sont des nomenclatures actives, puisque leur utilisation correcte permet. au terme de ce que l'on appelle généralement un dialogur homme-machinc. de faire fonctionner des ensembles techniques complexes. Ce n'est pas le lieu ici de discuter de la possibilité d'une intelligence artificielle (qui est l'objet de l'épistémologie appliquée: Baudet $199(0 \mathrm{~b})$, mais il est certain que les langues informatiques sont des exemples qui me paraissent particulièrement clairs de la dualité éditologique-terminologique de toute démarche scientifique. Une langue informatique est d'abord une liste de termes. ces termes font partic d'un système (il existe une syntaxe: manière d'ecrire des phrases correctes), el l'utilisation de celle langue conduit à des résultats pragmatiques. Outre celte dimension terminologique (la plus évidente pour l'utilisateur). il y a une dimension éditologique qui réside dans le double fait que ces langues sont le résultat de travaux d'équipe. el que ces langues sont soumises a des normalisations contrôles sociaux. mais par un sous-ensemble du corps social: la communauté informatique internationale). La science - discursive - se transforme en technologic - opératoire. Le réel n'est plus uniquement décrit à l'aide de termes. il est manipulé à l'aide de termes. Fi parce qu il y a un continuum science-technologie-industrie, on voit ici apparaître dans toute leur vigueur les considérations économiques: de puissantes multinationales jouent un rôle important dans la communauté informatique internationale qui tixe les standards en matière de génie logiciel. Mais on avait déjà vu que même dans le domaine de la recherche la plus fondamintale'. l'intérêt personnel du chercheur était bien présent.

\section{QUELQUES RESULTATS}

La prise en compte de la nature scientifique de la technique el de l'industrie nous paraît un des résultats majeurs de l'éditologie. Il y a interpénétration des activités de recherche purce de recherche appliquée. ce que l'examen critique des mécanismes d'édition rend évident (subsidiation de revues scientifiques par les milieux industriels: la question des brevets. qui sont des modalités d'édition tout à fait particulières è qui montrent le lien inextricable entre intérêt socio-économique. communication el développement du savoir). Le terme STI - science-technique-industric - est de plus en plus employé, et l'on peut espérer que les chercheurs en épistémologie continueront de sien servir pour éviter les fícheusés connotations idéologiques attachées au mot science, surtout quand il est utilisé de manière absolue: la Scionce' (On pourrait aussi bien employer technologie ì la place de STI. comme le montre cette excellente analyse (Guespin 1991: 76):

Liessentiel est que. à côté de sa fonction de connaissance théorique du monde, la science est désormais chargée d'une fonction d'amélioration de lat technique. El ceesl pour désigner ce nouveau moxle de coexistence. celte mise en rapport systématique de la science el de la kechnique, qu'a été créc le kerme de lechnologie. Toutefois, d'un point de vue philosophique. on peut penser que ces deux fonctions $n$ 'en font qu'une: on ne connaît qu'en transformant fnous ajouterions: et qu'en nommant]. et donc la connaissance scientifique du monde entièrement humanisé, socialisé, où nous vivons. ne peut progresser sans transformation constante de nos moyens d agir sur lui par les technique:s. 
Un deuxième résultat que l'éditologie est en droit de revendiquer est un renouveau d'intérêt pour les questions liées à la iulgarisation scientifíque'. C'est ainsi que, partant de l'éditologie. François Gaudin (1991: 136) a bien vu que la linguistique (et plus précisément ce qu il appelle socioterminologie) a un rôle à jouer dans la prise de conscience de la nature des obstacles auxyuels se heurte le corps secial dans la communication du savoir: lat langue "engage l'ensemble de nos conceptions du rapport entre la science et la société». Allant jusqu à proposer une écologie linguistique pour "faciliter la mise en moss" des acquisitions majeures de la STI. Avec pour objectif - - el l'on peut dire qu'ici l'éditologie. ce qui en montre bien la nature philosophique, débouche sur une éthique de tenter de construire les bases d'une authentique "démocratie cognitive" (Gaudin 1993: 216).

Un troisième résultat de l'éditologie serait d’apporter idées et moyens d'action à ceux que préccupe le sort des diverses langues européennes (dont le français) face à la domination de plus en plus pesante de l'anglo-américain dans la communication scientifique, technique et industrielle. ("est par l'étude de la communication scientifique dans toutes ses dimensions que l'on pourra comprendre pourquoi l'anglo-américain l'emporte. et que l'on pourra peut-être élaborer des stratégies défensives. kci, on est encore loin de pouvoir établir un bilan positif. D'une part, les politiques ou ne voient pas le problème. ou renâclent à le traiter de manière scientifique. $D$ autre part. les scientifiques admettent difficilement de placer sur un même plan leur travail de pénséce et l'activité technoindustrielle. ("est pourtant cette équivalence de nature épistémologique entre science et industrie qui est le sens de l'éditologice et l'on peut voir dans cetle réticence une preuve supplémentaire du refoulement de la nature sociale de la science chez ceux qui la font. Mais en se constituant en sociolinguistique de la science. l'éditologie ne peut pas refuser d'observer les comportements des hommes de science comme ils sont. de reconnaitre que l'expression de Ferruccio Rossi-Landi, yui parlait du langage, peut aussi s'appliquer à lat science: "la science comme travail el comme marché". qu'il y a. dans la coexistence potenticlle de plusicurs langues pour l'expression scientifique et dans le choix qui est fait linalement, des questions trèn simples d'intérêt personnel. et que, ches les scientifiques comme dans les autres groupes sociaux. cest bien la structure sociale qui détermine les comportements linguistiques. Les rapports sociaux, même chez les scientifiques. conditionnent les discours.

\footnotetext{
Notes

1. APPS. Assciation pour la promotion des publications xientutiques en langue française, fondée a Bruxelles an $|9 x|$.

2. Citom surtout Karl Mannhem en (iecorges (iurvitch. On peut y rattacher les travaux de Pierre Bourdien. quand il montre comment le haut lieu de la science yu en l'enseignement superieur ne fiat que recomstruire

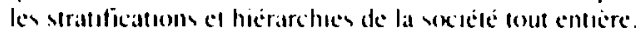

\section{RFFERFN('ES}

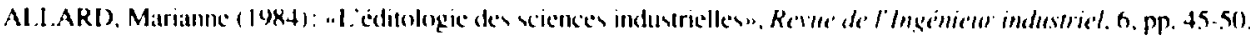

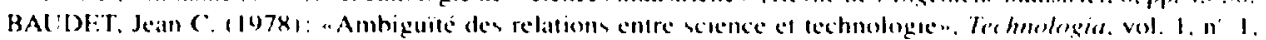
p). $17-201$

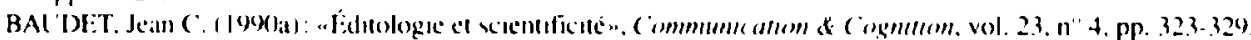

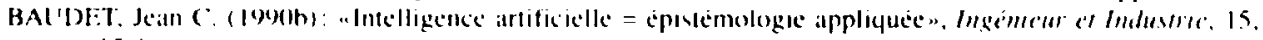
Pp. 1.5-16.

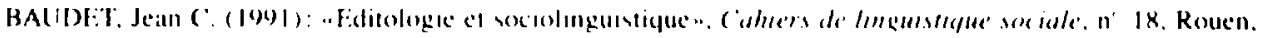
Universti: de Rouen. pp. $x \mid-(x)$.

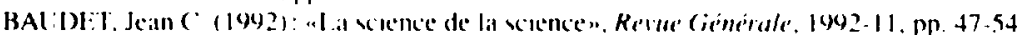

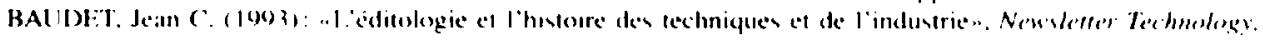

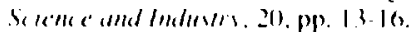




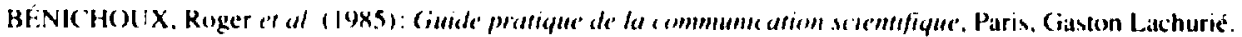

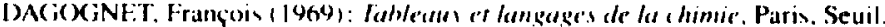

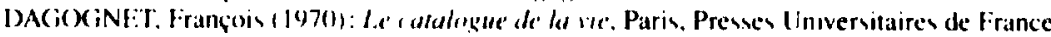

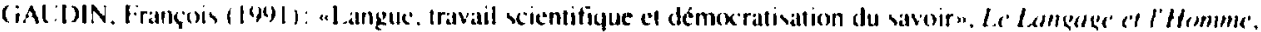
vol. $26.112-3 . \mathrm{pp} .129-1.39$

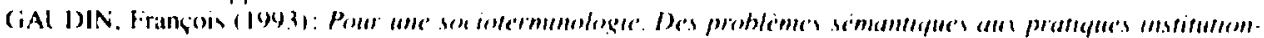
melle's, Roucon. Publicalliom de l'l'niversté de Rouen.

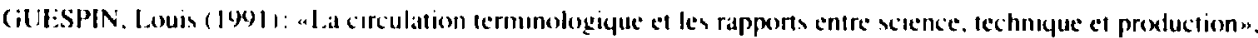

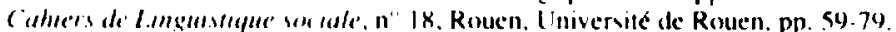

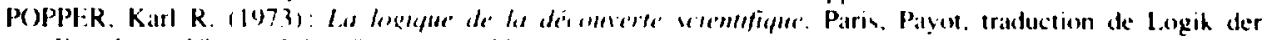
Forschung. Vienne. Juluus Sprongerer. I435. 